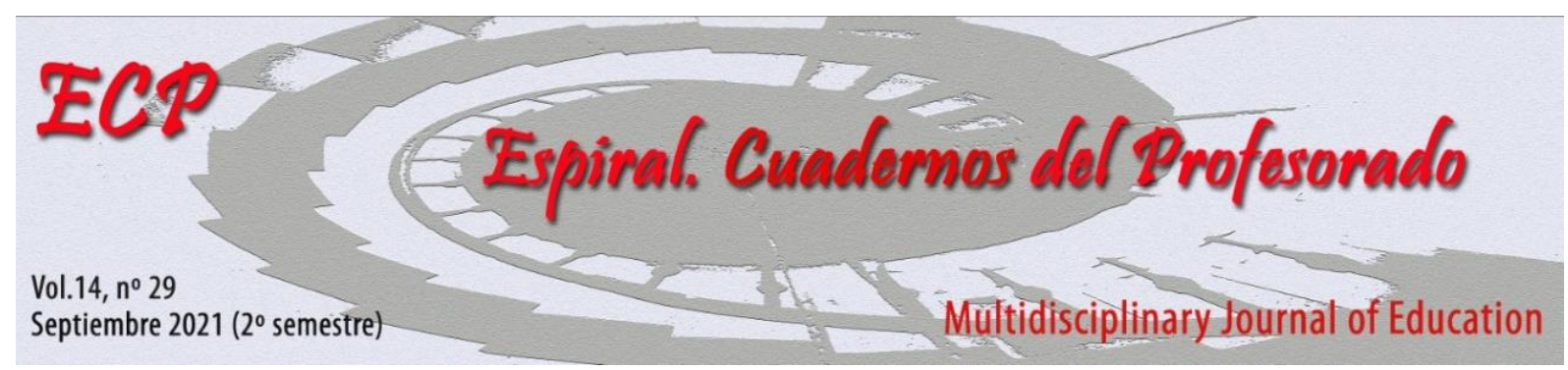

\title{
Análisis del movimiento durante la escalada como estrategia para el aprendizaje de la anatomía del aparato locomotor en Ciencias del Deporte
}

\section{Analysis of movement during climbing as a strategy for learning the anatomy of the locomotor system in Sport Sciences}

\author{
Raúl Baños ${ }^{1,2}$, Ángel Toval-Sánchez ${ }^{3}$, Nicanor Morales-Delgado ${ }^{4}$, \\ y José Luis Ferrán ${ }^{3}$
}

\footnotetext{
${ }^{1}$ Facultad Ciencias de la Actividad Física y del Deporte-INEF, Universidad Politécnica de Madrid, España;

${ }^{2}$ UABC-CA-318 Universidad Autónoma de Baja California, México; ${ }^{3}$ Departamento de Anatomía Humana y Psicobiología. Facultad de Medicina. Universidad de Murcia, España; ${ }^{4}$ Departamento de Histología y Anatomía. Facultad de Medicina. Universidad Miguel Hernández, España.
}

\section{Resumen}

El uso de las tecnologías de la información y la comunicación permite crear recursos didácticos que facilitan el aprendizaje fundamentalmente por su efecto motivacional. El objetivo del estudio ha sido analizar el uso de videos y de imágenes estáticas de escalada, en el proceso de enseñanza-aprendizaje del aparato locomotor en la asignatura Anatomía Funcional en Ciencias de la Actividad Física y del Deporte (CAFD). Un total de 106 estudiantes del grado de CAFD completaron el presente trabajo. Para el análisis del efecto en el aprendizaje se ha realizado un examen que contenía cinco preguntas sobre la anatomía aplicada a la escalada tras la visualización de los vídeos/imágenes. Tras el análisis estadístico, se observó un porcentaje de aciertos de 66,9\% y 69,2\% en las preguntas 1 y 3 respectivamente, mientras que en las preguntas 2, 4 y 5 se observó un porcentaje de aciertos inferior. Esta metodología se basa en un análisis del movimiento que permite una comprensión funcional de la anatomía del aparato locomotor en actividades deportivas específicas.

Palabras clave: Anatomía del movimiento; Anatomía aplicada; Anatomía para deportistas; Anatomía CAFD.

\begin{abstract}
The use of information and communications technologies allows the creation of teaching resources that facilitate learning, mainly due to their motivational effect. The study aimed to analyze the use of videos and static images of climbing in the teaching-learning process of the locomotor system in the Functional Anatomy signature in Physical Activity and Sport Science (CAFD). A total of 106 students from the CAFD degree completed this work. For the analysis of the effect on learning, an exam containing five questions on the anatomy applied to climbing was carried out after viewing the videos/images. After the statistical analysis, a percentage of correct answers of $66.9 \%$ and $69.2 \%$ was observed in questions 1 and 3, respectively, while in questions 2, 4 and 5, a lower percentage of correct answers were observed. This methodology is based on an analysis of the movement that allows a functional anatomical understanding of the locomotor system in specific sports activities.
\end{abstract}

Keywords: Anatomy of movement; Applied anatomy; Anatomy for athletes; Anatomy CAFD.

Fecha de recepción: 01/02/2021

Fecha de aceptación: 24/03/2021

Correspondencia: José Luis Ferrán, Facultad de Medicina, Universidad de Murcia, España Email: jlferran@um.es 


\section{Introducción}

La actual crisis del COVID-19, ha determinado numerosos cambios en la vida cotidiana de los seres humanos, viéndose afectado también el sistema educativo y los procesos de enseñanza-aprendizaje. Aunque desde hace años la literatura científica hacía hincapié en la importancia de evolucionar hacia la enseñanza en línea (Miarso, 2004), la actual pandemia de COVID-19, ocasionada por el virus SARSCoV2, ha obligado a acelerar dicho proceso (Crawford et al., 2020). De esta forma, la enseñanza presencial en las universidades se ha visto desplazada por la educación en línea, haciendo uso de las plataformas elearning (Flores, Correa, \& Cervantes, 2020; Srinivasan, 2020). Este sistema, es un proceso educativo en el que el aprendizaje es apoyado por recursos mediáticos, involucrando a la red de comunicación internet, y a otras tecnologías o medios como la tecnología multimedia, vídeo, audio, comunicación por satélite, etc., siendo estas tecnologías aplicadas de forma sincrónica y asincrónica (Hayashi, 2020).

En esta línea, la enseñanza de la anatomía también se ha visto obligada a evolucionar hacia la enseñanza virtual (Evans et al., 2020; Ravi, 2020). El estudio de la anatomía tiene diferentes enfoques como, por ejemplo, la anatomía aplicada al movimiento que se imparte en el grado de Ciencias de la Actividad Física y del Deporte (CAFD). El grado en CAFD, estudia gran diversidad de contenidos relacionados con una gran variedad de deportes, entre los que son objeto de análisis los deportes tradicionales como el fútbol, baloncesto, atletismo, etc... Sin embargo, los contenidos relacionados con las actividades físicas en el medio natural (escalada, rapel, orientación...), van generando cada vez más interés entre los estudiantes, resultando más motivante el aprendizaje de dichas disciplinas (BaenaExtremera \& Granero-Gallegos, 2013a; Granero \& Baena, 2011).

Para comprender la anatomía y biomecánica del aparato locomotor, la escalada es un deporte idóneo como herramienta de aprendizaje. En el proceso de enseñanza y aprendizaje de la asignatura Anatomía Funcional, resultan esenciales aquellos deportes que permitan observar durante su práctica la complejidad y rangos de movimiento que involucran especialmente a las extremidades del aparato locomotor. Esto permite un mejor análisis y comprensión del movimiento generado por los músculos que cruzan los diferentes complejos articulares En este sentido, estudios previos han sugerido que la aplicación de esta metodología durante la práctica de la escalada resulta ser una herramienta clave en el aprendizaje de la asignatura Anatomía en CAFD (Baños et al., 2015; Ferrán 2015).

El uso de las tecnologías de la información y la comunicación (TIC) en el proceso de enseñanzaaprendizaje va adquiriendo cada vez más un rol destacado. En el ámbito de CAFD, los docentes utilizan predominantemente las TIC para fines administrativos tales como el registro y presentación de informes de los estudiantes (Thomas \& Stratton, 2006), sin llegar a potenciar el enorme papel pedagógico que aportan las herramientas digitales. Dentro de las TIC, el blog o bitácora es una herramienta digital que ofrece la posibilidad de actualizarlo periódicamente y estructurarlo en función del criterio de los autores docentes. Tal y como afirman Prieto, Smok y Rojas (2011), entre sus características destacan la posibilidad de dirigirse a gran cantidad de personas, con o sin conocimientos del ámbito, sin necesidad de que el docente disponga de elevados conocimientos técnicos, siendo sencillo de administrar y pudiendo resolver las dudas presentadas por los internautas. Además, según diversos estudios los estudiantes perciben que los blogs promueven una mayor colaboración, diálogo y participación activa (Hemmi, Bayne \& Tierra, 2009; Hsu, 2008; Oliver \& Maíz, 2008). Hastie, Casey y Tarter (2010) destacan que el uso de los blogs en la materia de Educación Física conduce a una experiencia de aprendizaje más significativo comparado al obtenido empleando las metodologías tradicionales, debido a que la preparación de las próximas sesiones puede tener un carácter más anticipativo y colaborativo con el alumnado. Boulos y Wheeler (2007) definen como inteligencia colaborativa al proceso mediante el cual los alumnos van participando de forma activa en el blog, con un compromiso serio y constante por parte del alumnado, pudiéndose producir una serie de conversaciones en la que van diseñando y construyendo su propio aprendizaje de la asignatura.

La relevancia y el impacto en la innovación educativa que tiene el uso de los blogs como herramientas pedagógicas en el proceso de aprendizaje de los estudiantes universitarios está adquiriendo progresivamente mayor importancia. Por ello, en la asignatura Anatomía Funcional de la Universidad de Murcia, se ha creado un blog específico de la asignatura con sus respectivas cuentas de YouTube, Twitter y Facebook como herramientas didácticas digitales. Así, el objetivo del presente estudio ha sido analizar

Espiral. Cuadernos del Profesorado | ISSN 1988-7701 | 2021, 14(29), 102-112 
el conocimiento anatómico del aparato locomotor alcanzado por los alumnos de CAFD durante la ejecución de movimientos de escalada, utilizando para el aprendizaje videos e imágenes estáticas gestionadas a través del blog.

\section{Método}

\section{Diseño del Estudio}

Se utilizó un diseño no experimental, descriptivo y transversal. Participaron en el estudio un total de 106 estudiantes, 78 hombres y 28 mujeres, matriculados en la asignatura de Anatomía Funcional en el grado de CAFD de la Universidad de Murcia, con edades comprendidas entre 19 y 49 años (21,6 $\pm 4,7$ años) participaron en el presente estudio.

\section{Instrumentos}

Para evaluar los conocimientos adquiridos se incluyeron 5 preguntas sobre el análisis del movimiento durante la escalada en el examen final de la asignatura, que estaba compuesto por un total de 60 preguntas tipo test, de elección múltiple y con una sola respuesta válida. El examen fue preparado por el coordinador de la asignatura con una duración total de 90 minutos para responder a la totalidad de los cuestionarios. Las preguntas relacionadas con la modalidad deportiva de escalada en base a la Figura 1 fueron las siguientes:

1) Pregunta 1 (punto 1 de la imagen). Indicar la opción CORRECTA en relación a la articulación y región indicadas. Posibles respuestas: A) En la rotación observada participa el supraespinoso. B) El dorsal ancho permite la retroversión del hombro. C) El trapecio porción inferior es aductor del brazo. D) El ligamento trapezoide estabiliza la articulación esterno-costo-clavicular.

2) Pregunta 2 (punto 2 de la imagen): Indicar cuál de los siguientes músculos NO participa en la posición observada en la articulación indicada (codo). Posibles respuestas: A) Extensor radial largo del carpo. B) Palmar largo. C) Flexor común profundo de los dedos. D) Flexor radial del carpo.

3) Pregunta 3 (punto 3 de la imagen): Indicar la opción INCORRECTA sobre la extremidad indicada. Posibles respuestas: A) El aductor menor favorece la posición observada. B) El glúteo mayor favorece la posición observada. C) El cuádriceps femoral favorece la posición observada. D) El popliteo se opone a la posición observada.

4) Pregunta 4 (punto 4 de la imagen): Indicar la opción INCORRECTA sobre la articulación indicada. Posibles respuestas: A) El gemino inferior se opone a la rotación observada en la cadera. B) El semimembranoso favorece la posición observada en la cadera. C) El glúteo menor favorece la posición observada en la cadera. D) El pectíneo se opone a la posición observada en la cadera.

5) Pregunta 5 (punto 5 de la imagen): Indicar la opción CORRECTA sobre la región y articulación indicadas. Posibles respuestas: A) Los lumbricales están en un plano profundo. B) El abductor del dedo pequeño se encuentra en un plano profundo. C) El extensor corto de los dedos extiende las falanges proximales. D) El flexor corto del dedo gordo se encuentra en un plano superficial. 


\section{Figura 1.}

Imagen sobre escalada visualizada en el examen final de Anatomía Funcional (Fuente: Imagen extraída del blog https://functionalanatomysite.wordpress.com/).

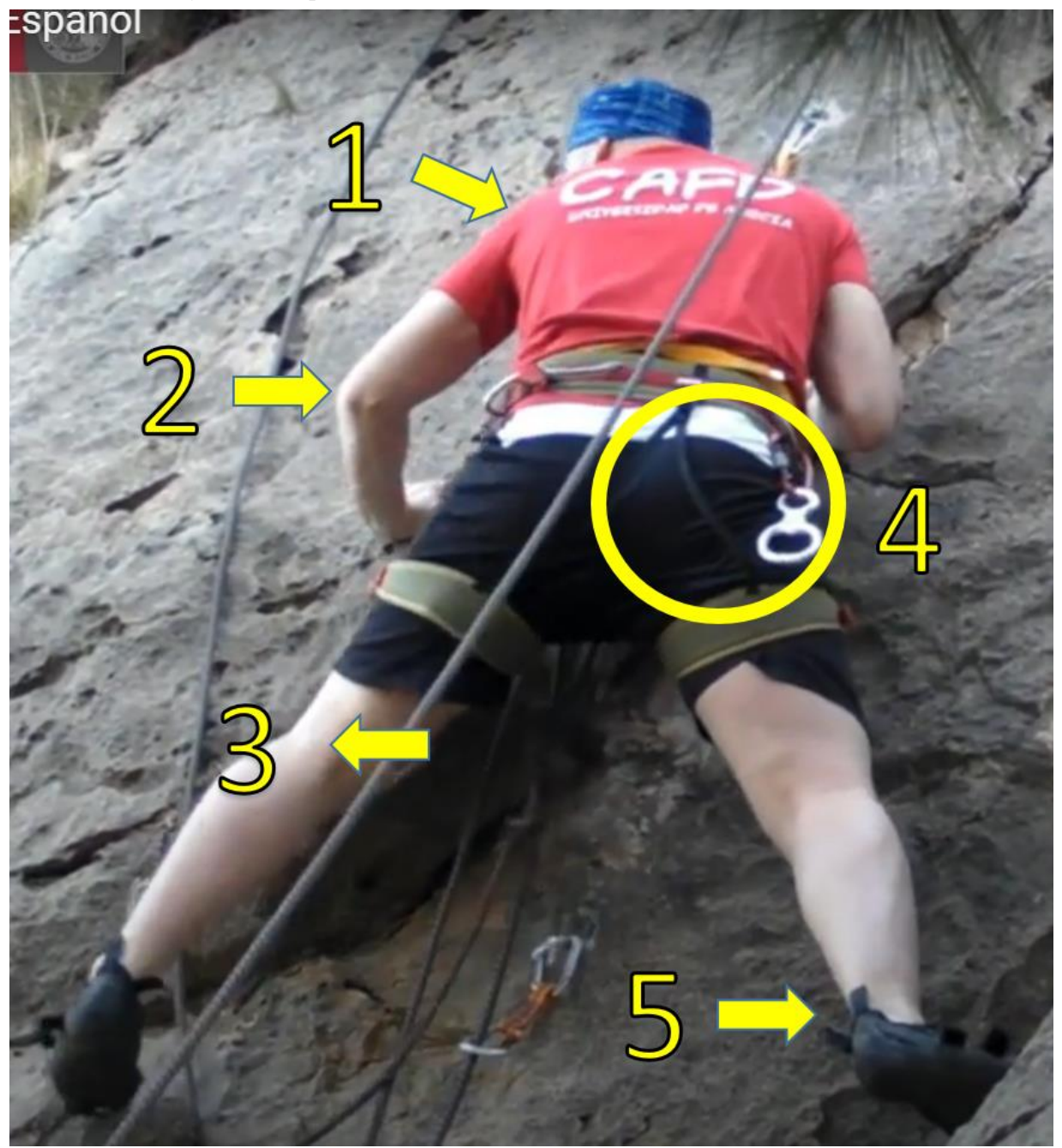

\section{Procedimiento}

Todos los estudiantes, recibieron los mismos contenidos de Anatomía Funcional, incluidos en la guía docente, con una duración de cuatro meses y una carga lectiva de 6 créditos ECTS. Siguiendo la guía docente, se impartieron 3 horas lectivas semanales de clase teórica presencial mediante el formato de presentación PowerPoint y una 1 hora lectiva semanal de clase práctica.

El Grupo de Innovación Docente del Departamento de Anatomía Humana de la Universidad de Murcia diseñó un blog como recurso didáctico, que utiliza principalmente vídeos de distintos deportes (https://functionalanatomysite.wordpress.com/). Inicialmente, se presentó a los estudiantes en clase el funcionamiento del blog, indicando las posibilidades de aprendizaje que permitía dicho recurso, siendo estos recursos utilizados tanto en clases presenciales como de forma autónoma por el propio estudiante. De esta manera, se ofreció a los estudiantes la posibilidad de evaluar la comprensión de los contenidos impartidos en la asignatura de Anatomía Funcional aplicándolos al deporte de escalada en un contexto real. La forma en que el alumnado podía autoevaluarse mediante el blog era la visualización de vídeo e imágenes estáticas seleccionadas. El vídeo se detenía durante unos segundos en algún momento clave de la acción técnico-deportiva, momento en el cual se hacía una pregunta sobre los contenidos relacionados con los movimientos anatómicos y los complejos articulares involucrados durante la ejecución de la acción 
por el escalador. Finalmente, se evaluaron los contenidos adquiridos por los estudiantes a través de un examen teórico tipo test.

\section{Análisis Estadístico}

Tras la codificación y tabulación de los datos, se realizó un análisis descriptivo de las frecuencias, el cual ha sido seleccionado por su orientación en el campo sociológico, y ha posibilitado la puesta en práctica de la técnica estadística precisa para el presente estudio. El análisis estadístico fue realizado mediante el paquete estadístico SPSS (Statistical Package for Social Sciences, v. 20.0, para Windows; SPSS Inc, Chicago).

\section{Resultados}

\section{Porcentajes de aciertos y errores}

En la Figura 2 se observa que el porcentaje de aciertos es mayor que el porcentaje de errores para las preguntas 1 y $3(61,3 \%$ y 64,2\% respectivamente), mientras que en las preguntas 2,4 y 5 , el porcentaje de preguntas incorrectas es mayor que el de las respuestas correctas; siendo estos valores del $67,0 \%, 79,2 \%$ y $69,5 \%$, respectivamente.

\section{Figura 2.}

Porcentaje de aciertos y errores del examen tipo test.

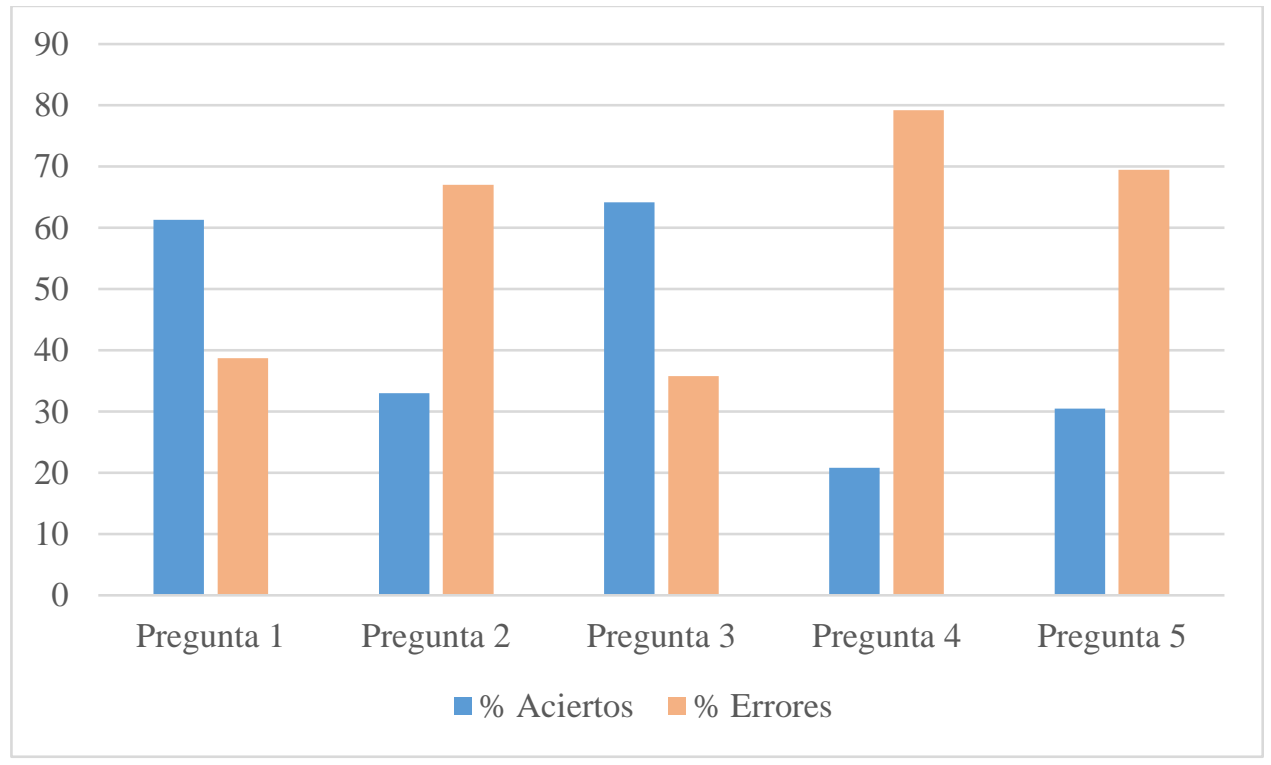

\section{Relación de respuestas pregunta 1}

Tal y como se puede observar en la Figura 3, el 61,3\% de los estudiantes eligió la respuesta correcta "El dorsal ancho permite la retroversión del hombro", mientras que un 21,7\% eligieron la opción C "El trapecio porción inferior es aductor del brazo", un 9,4\% la respuesta A "En la rotación observada participa el supraespinoso" y un 6,6\% la opción D "El ligamento trapezoide estabiliza la articulación esterno-costo-clavicular". 


\section{Figura 3.}

Opciones de respuesta seleccionada en la pregunta 1.

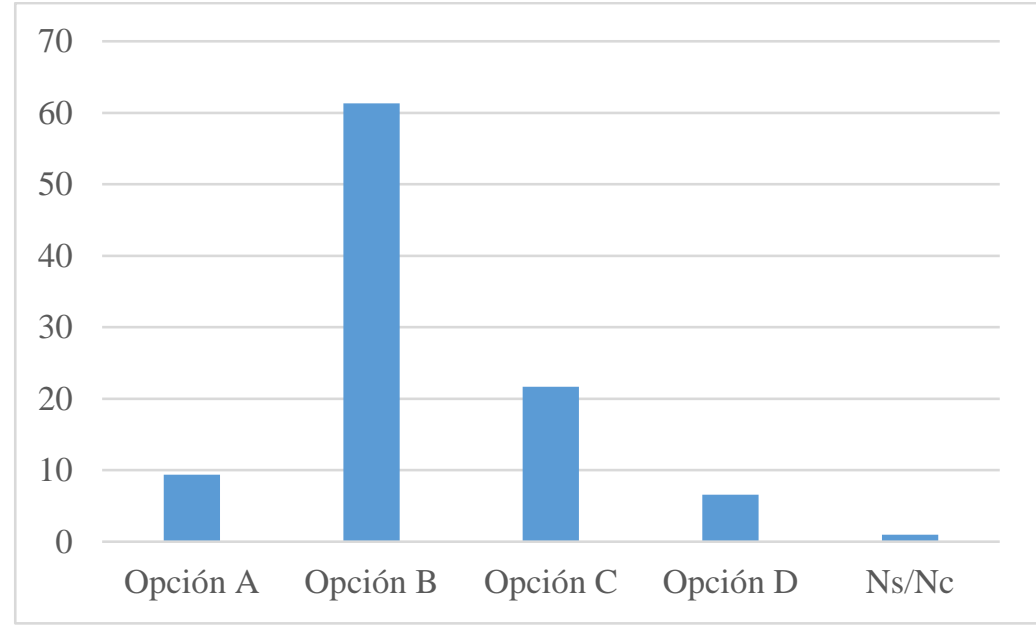

\section{Relación de respuestas pregunta 2}

En la pregunta 2, el 38,7\% de los estudiantes erró y eligió la opción A "Extensor radial largo del carpo". La opción correcta era la C "Flexor común profundo de los dedos", pero solo la seleccionaron el $33,0 \%$ de los participantes; el resto, un 14,2\% y un 13,2\%, seleccionaron "Palmar largo" y "Flexor radial del carpo" respectivamente, tal y como se muestra en la Figura 4.

Figura 4.

Opciones de respuesta seleccionada en la pregunta 2.

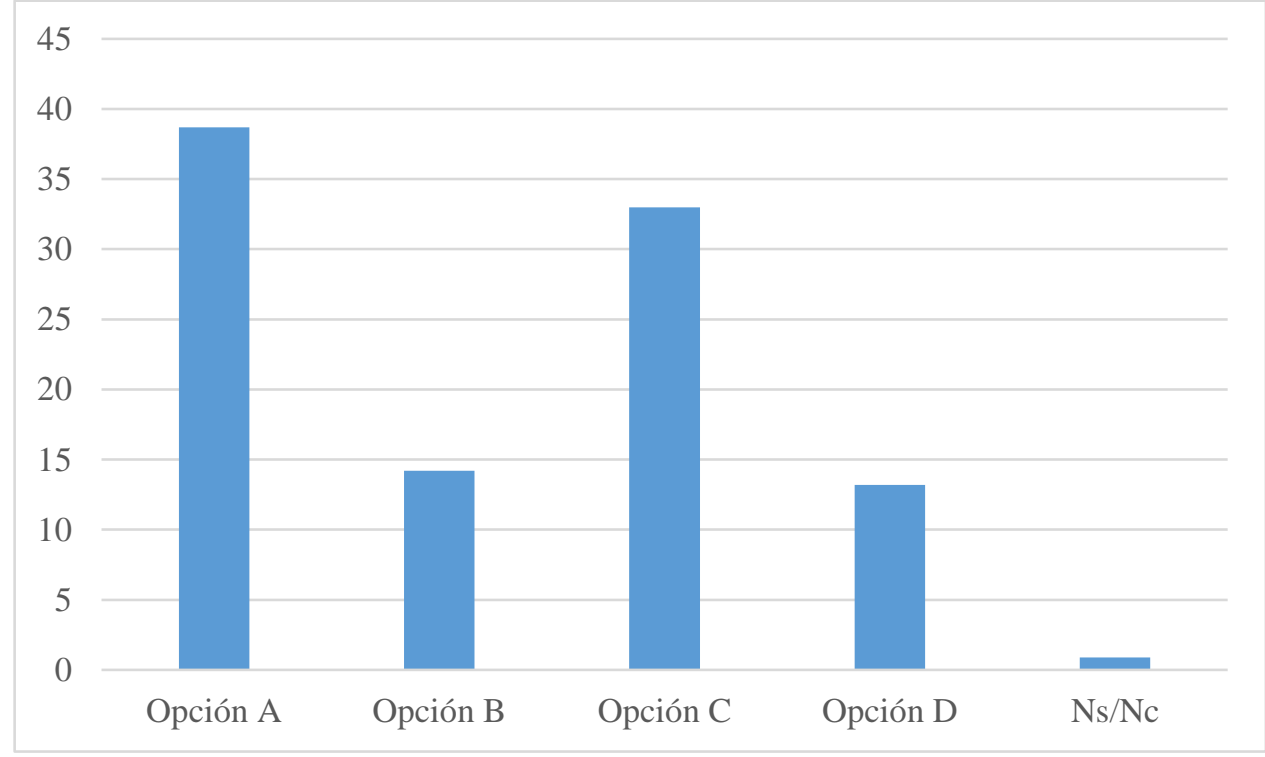

\section{Relación de respuestas pregunta 3}

En la pregunta 3, la mayoría de los discentes $(64,2 \%)$ seleccionó la opción válida "El aductor menor favorece la posición observada", seguida de la opción C "El cuádriceps femoral favorece la posición observada" con un $17,9 \%$, mientras que una pequeña proporción de estudiantes eligió la opción D "El poplíteo se opone a la posición observada" con un 9,4\%, y un 8,5\% la opción B "El glúteo mayor favorece la posición observada” (Figura 5). 
Análisis del movimiento durante la escalada como estrategia para el aprendizaje de la anatomía del aparato locomotor en Ciencias del Deporte

\section{Figura 5.}

Opciones de respuesta seleccionada en la pregunta 3.

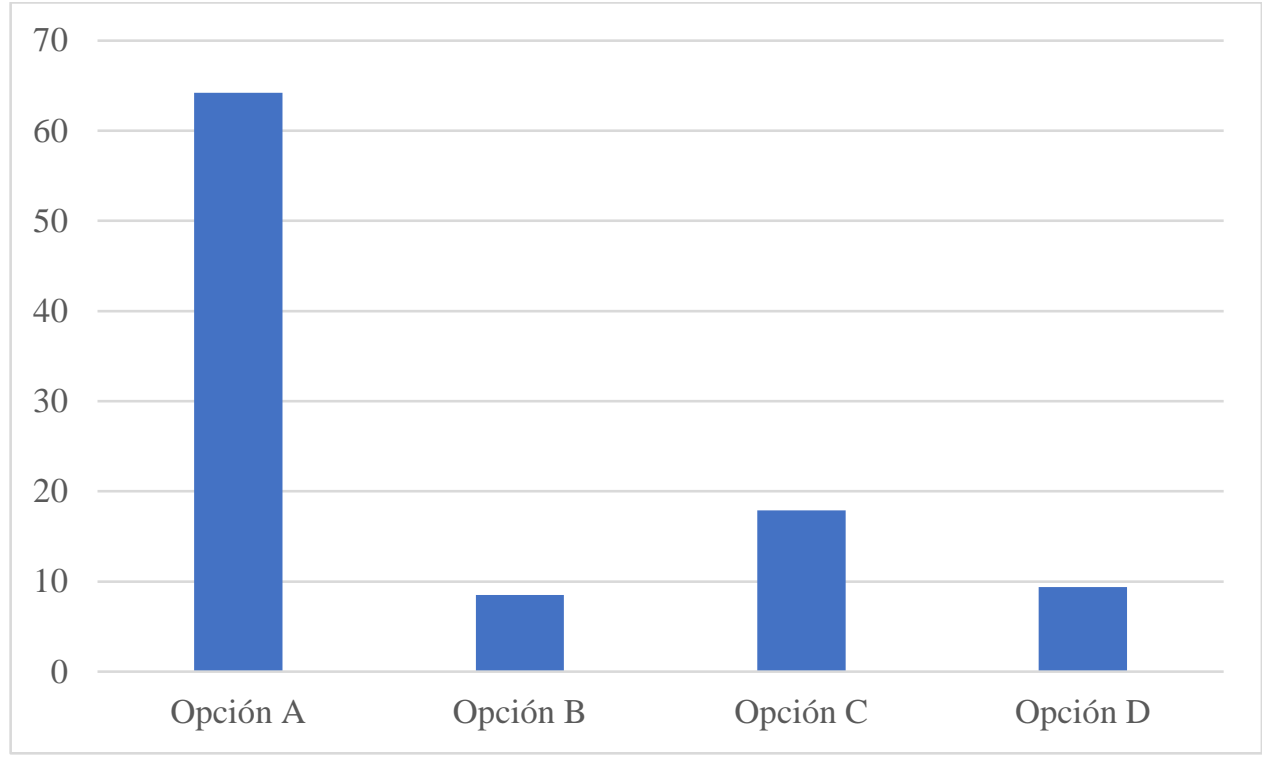

\section{Relación de respuestas pregunta 4}

En la pregunta 4, tan solo un 20,8\% de los evaluados eligió la opción correcta "El gemino inferior se opone a la rotación observada en la cadera". Un 48,1\% optó erróneamente por "El semimembranoso favorece la posición observada en la cadera". Además, un 24,5\% eligió la opción D "El pectíneo se opone a la posición observada en la cadera" y, por último, un 5,7\%, la respuesta C "El glúteo menor favorece la posición observada en la cadera (Figura 6).

\section{Figura 6.}

Opciones de respuesta seleccionada en la pregunta 4.

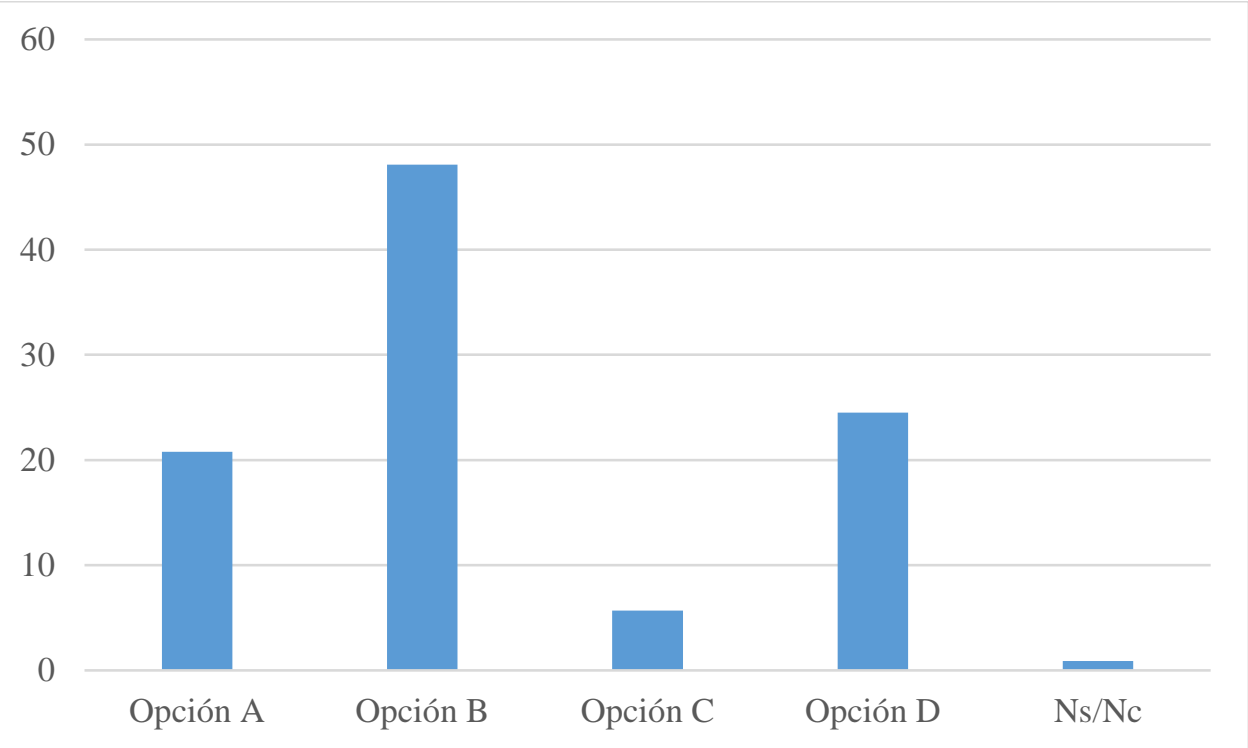

\section{Relación de respuestas pregunta 7}

Por último, en la Figura 7 se muestra que los resultados de la pregunta 5 fueron muy similares, excepto para la opción B "El abductor del dedo pequeño se encuentra en un plano profundo", que fue elegida por un $8,5 \%$ del alumnado. Con un porcentaje de $32,1 \%$ se seleccionó la respuesta A "Los 
lumbricales están en un plano profundo", mientras que las opciones C "El extensor corto de los dedos extiende las falanges proximales" y D "El flexor corto del dedo gordo se encuentra en un plano superficial" estuvieron muy igualadas, con un $30,2 \%$ y $29,2 \%$, respectivamente

Figura 7.

Opciones de respuesta seleccionada en la pregunta 7.

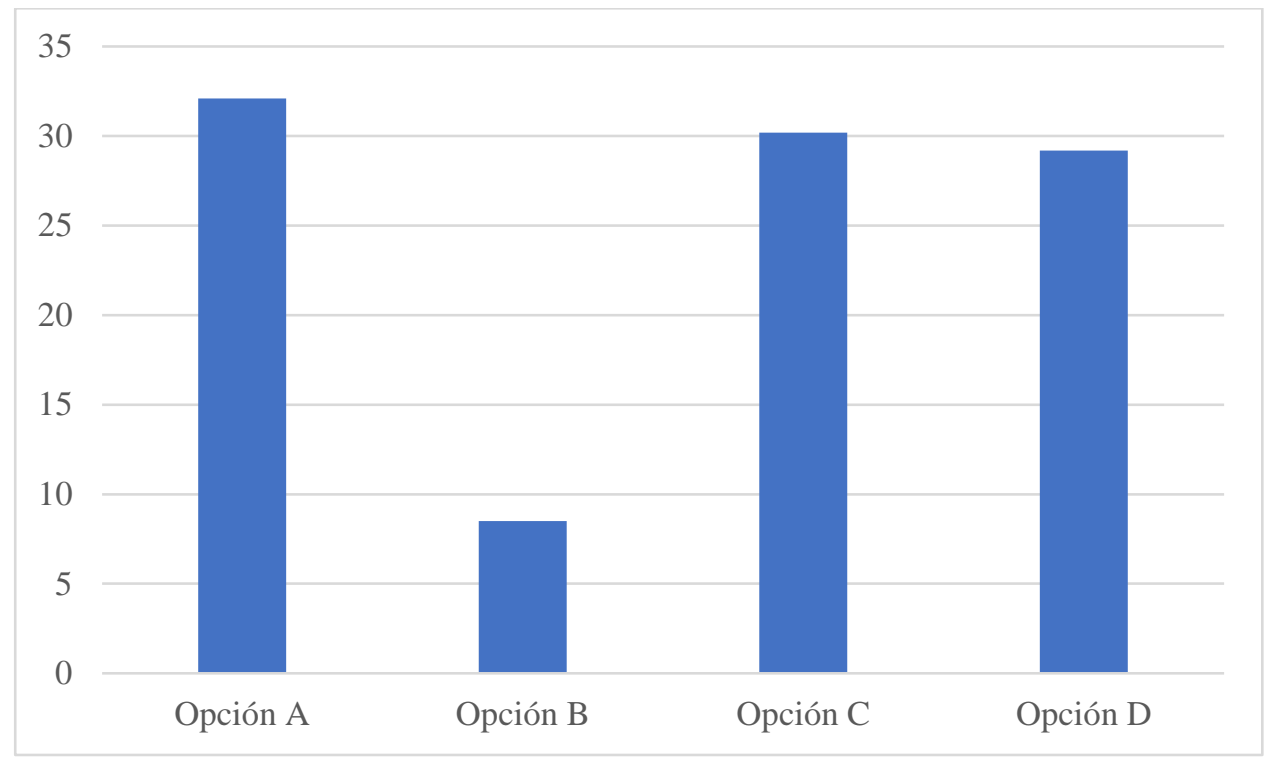

\section{Discusión}

Son escasas las investigaciones que relacionan el aprendizaje de la Anatomía del aparato locomotor de forma directa con el análisis del movimiento durante la práctica de un deporte. Para el Espacio Europeo de Educación Superior (EEES) constituye un verdadero reto en la enseñanza universitaria lograr que el conocimiento que adquieran los estudiantes tenga una aplicación inmediata en el ámbito laboral. Teniendo como fin la mejora del aprendizaje de la anatomía, hemos creado recursos digitales para facilitar la adquisición de conocimientos por parte del estudiante. Así, el objetivo de este estudio fue analizar el conocimiento anatómico del aparato locomotor alcanzado por los alumnos de CAFD durante la ejecución de movimientos de escalada, utilizando para el aprendizaje vídeos e imágenes estáticas gestionadas a través del blog.

En las preguntas 1 y 3 se obtuvo un elevado porcentaje de aciertos (61,3\% y 64,2\%, respectivamente) en comparación con los estudiantes que seleccionaron una opción errónea, un hecho que indica además la baja dificultad que tiene para el estudiante la aplicación de este tipo de conceptos anatómicos. Un proyecto similar elaborado por la Universidad de Granada, (https://www.ugr.es/ dlcruz/) afirma la necesidad de transversalidad entre distintas disciplinas (anatomía, voleibol y fútbol), que están aparentemente separadas en su contexto científico y didáctico. Como diferencia significativa, nuestra metodología podría interpretarse como más aplicada para la comprensión del aparato locomotor en movimiento, puesto que mediante vídeos e imágenes se desarrollan actividades en contextos totalmente deportivos, al contrario de la anterior, que se basa en publicar contenidos anatómicos en la red desde un punto de vista y marco puramente teórico.

En contraposición, se observa un escaso porcentaje de aciertos (inferior al 33\%) en las preguntas 2, 4 y 5. En cuanto a la pregunta 2, la opción a elegir implicaba un alto nivel de conocimiento, estando dentro del examen del grupo del $15 \%$ de preguntas más complejas, implicando por parte del alumno tener o no el conocimiento sobre la acción específica que ejercen estos músculos sobre el codo cuando su función predominante en alguno de ellos es sobre el carpo o las falanges. En cuanto a las preguntas 4 y 5 , también se evaluaba un alto nivel de conocimiento y además la habilidad de pensar los conceptos de forma indirecta, ya que se preguntaba por lo incorrecto en torno a funciones que no se suelen destacar con frecuencia con respecto a esta musculatura. Ambas preguntas también formaban parte del grupo de $15 \%$ 
de preguntas de gran dificultad que habitualmente tienen un nivel de respuesta más bajo que el aquí obtenido. Por tanto, el menor grado de acierto que hemos registrado podría estar vinculado a la complejidad del concepto evaluado. Además, estos datos sugieren que los conceptos evaluados en estas preguntas resultan más difíciles de aplicar por parte del alumno produciendo un mayor rango de error. Este aspecto está siendo abordado actualmente por el grupo bajo el diseño de 5 niveles de complejidad anatómica que serán evaluados bajo esta misma estrategia. De esta forma se podrá valorar cuál es el grado de aprendizaje en cada nivel de complejidad planteado y se pretende además que el alumno pueda adquirir habilidades para resolver de forma más exitosa la aplicación de este tipo de dificultades conceptuales.

Debido a la escasez de estudios que relacionen la aplicación de las TIC en la enseñanza de Anatomía Funcional en torno a distintos deportes, es preciso discutir estos resultados con otras estrategias para la enseñanza de la anatomía en otros grados diferentes al grado de Ciencias de la Actividad Física y del Deporte. Los resultados del presente trabajo son similares a los encontrados por Fonseca y Fonseca (2010) en el área de la anatomía patológica. En su estudio la metodología de enseñanza consistió en la realización de diagnósticos mediante la observación de imágenes en estudios universitarios, que según los autores fue muy novedosa y de gran interés entre los estudiantes. También, en el área de la anatomía patológica, se confirmó que la microscopía virtual como uso de herramientas pedagógicas digitales puede reemplazar de forma efectiva los métodos tradicionales de aprender patología (Alós et al., 2015). Por otra parte, en el campo del estudio de la anatomía de la placenta, otros autores han concluido que el blog permite a los docentes contar con un modelo de aprendizaje personalizado y centrado en el estudiante (Prieto, Smok, \& Rojas, 2011).

Es interesante mencionar que otros estudios (Baena-Extremera \& Granero-Gallegos, 2013b) han investigado sobre la utilización del iBook como herramienta didáctica del estudio de la Anatomía en el área de la Educación Secundaria, donde los estudiantes encontraron un material novedoso e innovador. Sin embargo, continuaron impartiendo contenidos teóricos tradicionales sin llegar a aplicaciones prácticas en la enseñanza de la anatomía en el contexto deportivo, un aspecto que la propuesta del presente trabajo contribuiría a impulsar (Baena-Extremera \& Granero-Gallegos, 2013b). También en el área de la Educación Obligatoria, autores como Molina, Castillo, Rodríguez y Díaz, (2010) han resaltado que el aprendizaje de la anatomía aplicada a la Educación Física resulta más eficiente y duradero en el tiempo cuando se realiza desde una perspectiva práctica que si se imparte desde una perspectiva puramente teórica. Nuestra propuesta implica una aplicación de los contenidos teóricos anatómicos del aparato locomotor en un contexto real, para una mejor comprensión funcional de una actividad deportiva.

\section{Conclusiones}

El material digital creado por el Grupo de Innovación Docente en Anatomía de la Universidad de Murcia es una herramienta novedosa e interesante para el aprendizaje de la anatomía en un contexto deportivo y natural. Esta metodología se puede enmarcar como parte de la comprensión holística de la anatomía, permitiendo al estudiante una interpretación funcional durante la ejecución de la escalada de conceptos anatómicos puramente descriptivos. Es un modelo de enseñanza novedoso e innovador, por lo que se debe continuar elaborando más material didáctico de esta índole y perfeccionar el ya elaborado. De esta forma se anima a los anatomistas e instituciones a preparar material de buena calidad, que debería estar disponible en línea para los estudiantes (Raikos \& Waidyasekara, 2014).

Conocedores de algunas limitaciones del presente estudio, sería interesante para futuros trabajos analizar los perfiles psicológicos y/o la motivación del alumnado generada en los estudiantes (Baños, Barretos-Ruvalcaba, \& Baena-Extremera, 2019; Martínez-de-Ojeda, Méndez-Giménez, GutiérrezSánchez-Osorio, \& López-Delgado, 2020; Moreno, Zomeño, Marín, Ruiz, \& Cervelló, 2013), así como el rendimiento académico (Fernández, 2004) asociado al uso de estas metodologías.

Contribución de cada Autor: conceptualización, R.B, y J.F.; metodología, R.B, A.T., N.M. y J.F.; validación, R.B, y J.F.; análisis, R.B, A.T., N.M. y J.F.; escritura del borrador del manuscrito, R.B. y J.F.; escritura, revisión y edición, R.B, A.T., N.M. y J.F.; supervisión, J.F. 
Financiación: Esta investigación no recibió ninguna financiación externa.

Agradecimientos: El trabajo fue realizado en el marco de los proyectos de innovación docente 2014/2015 (Producción de material didáctico digital para el análisis del movimiento y comprensión de la anatomía en el Grado de Ciencias de la Actividad Física y Deporte) y 2015/2016 (Anatomía del movimiento en la enseñanza de grado en CAFD) de la Universidad de Murcia.

Conflicto de Intereses: Las/os autoras/es declaran que no tienen conflicto de intereses.

\section{Referencias}

Alós-i-Hernández, L., Ordi, O., Martínez Pozo, A., Ordi-i-Majà, J., Cuatrecasas-Freixas, M., Ramírez Ruz, J., ...\& Díaz Lorca, M. A. (2015). Microscopía virtual en la enseñanza de la Anatomía Patológica en Medicina. Pòsterpresentat a: Vuitenatrobada de professorat de Ciències de la Salut. Universitat de Barcelona. Facultat de Medicina.

Baena-Extremera, A., \& Granero-Gallegos, A. (2013a). Utilización de iBook en la Enseñanza de Contenidos de Anatomía en Educación Secundaria. International Journal of Morphology,31(2), 505-511. https://doi.org/10.4067/S0717-95022013000200024

Granero-Gallegos, A., \& Baena-Extremera, A. (2013b). El piragüismo en la formación permanente del profesorado como actualización de contenidos en Educación Física. Espiral. Cuadernos del Profesorado, 6(11), 311. https://doi.org/10.25115/ecp.v6i11.947

Baños, R., Morales, N., López-Iborra, L., Martínez-Armada, C., De la Cruz, E., Escribano, P., Moran, R., Pallares, J., Toval, A., Vera, J., Ferrán J.L. (2015). Análisis dinámico del movimiento en escalada, surf y slackline. Nuevas herramientas para la enseñanza de la anatomía en CAFD. En XXII Congreso Internacional de la Sociedad Española de Educación Médica. Universidad de Murcia.

Baños, R., Barretos-Ruvalcaba, M., \& Baena-Extremera, A. (2019). Protocolo de estudio de las variables académicas, psicológicas y de actividad física que influyen en el rendimiento académico de adolescentes mexicanos y españoles. Espiral. Cuadernos del Profesorado, 12(25), 89-99. https://doi.org/10.25115/ecp.v12i25.2480

Boulos, M.N.K., \&Wheeler, S. (2007). The emerging Web 2.0 social software: An enabling suite of sociable technologies in health and health care education. Health Information and Libraries Journal, 24, 2-23. https://doi.org/10.1111/j.1471-1842.2007.00701.x

Crawford, J., Butler-Henderson, K., Rudolph, J., Malkawi, B., Glowatz, M., Burton, R., ... \& Lam, S. (2020). COVID19: 20 countries' higher education intra-period digital pedagogy responses. Journal of Applied Learning \& Teaching, 3(1), 1-20. https://doi.org/10.37074/jalt.2020.3.1.7

De la Cruz, JC., Cueto, M., García, E., Rojas, J., De la Cruz, J. C., De la Cruz, A., (2014). Innovación en la gestión on-line en los procesos de enseñanza-aprendizaje en educación física. Propuesta interdisciplinar para la creación de una herramienta web. En L. M. Jiménez del Barco \& M. C. García (coord.), Innovación docente y buenas prácticas en la Universidad de Granada. Vol. 3 (pp. 61-72). Editorial Universidad de Granada.

Evans, D. J., Bay, B. H., Wilson, T. D., Smith, C. F., Lachman, N., \& Pawlina, W. (2020). Going virtual to support anatomy education: A STOPGAP in the midst of the Covid-19 pandemic. Anatomical Scieces Education, 13,279-283. https://doi.org/10.1002/ase.1963

Fernández, M. (2004). El clima motivacional de clase en estudiantes adolescentes de Buenos Aires. Revista Iberoamericana de Diagnóstico y Evaluación Psicológica, 18(2), 119-136.

Ferrán J.L. (2015). Hacia un enfoque moderno en la enseñanza de la anatomía funcional en CAFD. Priorizando el aprendizaje a partir de sus competencias específicas. En XXII Congreso Internacional de la Sociedad Española de Educación Médica. Universidad de Murcia.

Flores, G., Correa, M. E., \& Cervantes, M. G. (2020). Deporte, cultura y uso de tecnologías en el tiempo libre de jóvenes universitarios. Espiral. Cuadernos del Profesorado, 13(27), 144-150. https://doi.org/10.25115/ecp.v13i27.3491

Fonseca, G. M. \& Fonseca, A. C. (2010). Strategies of Education for the Study of Images in Pathology, School of Dentistry, Universidad Nacional de Cordoba, Argentina. International Journal of Morphology, 28(4):11131123. https://doi.org/10.1080/14759390903579133

Granero, A., \& Baena, A. (2011). Juegos y deportes de aventura en la formación permanente del profesorado. Revista Internacional de Medicina y Ciencias de la Actividad Física y el Deporte, 11(43), 531-547. Http://cdeporte.rediris.es/revista/revista43/artjuegos224.html.

Espiral. Cuadernos del Profesorado | ISSN 1988-7701 | 2021, 14(29), 102-112 
Análisis del movimiento durante la escalada como estrategia para el aprendizaje de la anatomía del aparato locomotor en Ciencias del Deporte

Hastie, P. A., Casey, A., \& Tarter, A. M. (2010). A case study of wikis and student-designed games in physical education. Technology, Pedagogy and https://doi.org/10.1080/14759390903579133

Hayashi, C. (2020). Tecnologias digitais na Educação a Distância: fases, modelos, plataformas e ferramentas. Research, Society and Development, 9(10), 1-30. https://doi.org/10.33448/rsd-v9i10.9295

Hemmi, A., Bayne, S., \& Land, R. (2009). The appropriation and repurposing of social technologies in higher education. Journal of Computer Assisted Learning, 25(1), 19-30. https://doi.org/ 10.33448/rsd-v9i10.9295

Hsu, J. (2008). Innovative technologies for education and learning: Education and knowledge-oriented applications of blogs, wikis, podcasts, and more. International Journal of Web-Based Learning and Teaching Technologies, 3(3), 62-81. https://doi.org/10.4018/jwltt.2008070106

Martínez de Ojeda, D., Méndez-Giménez, A., Gutiérrez Sánchez Osorio, M. L., \& López Delgado, A. (2020). Efectos del modelo TRIAL classroom en la autorregulación del alumnado de educación primaria frente a la metodología tradicional. Espiral. Cuadernos del Profesorado, 13(26), 86-97. https://doi.org/10.25115/ecp.v13i26.2689

Miarso, Y. (2004). Menyemai benih teknologi pendidikan. Yakarta.

Molina, J. J. M., Castillo, A. S., Rodríguez, A. I. L., \& Díaz, M. Z. (2010). Efecto de dos estilos de enseñanza (cognoscitivo versus tradicional) sobre el aprendizaje de conceptos de anatomía muscular en alumnos de Educación Física de $1^{\circ}$ de ESO. Apunts. Educación física y Deporte, (100), 23.

Moreno, J. A., Zomeño, T. E., Marín, L. M., Ruiz, L. M., \& Cervelló, E. M. (2013). Percepción de la utilidad e importancia de la educación física según la motivación generada por el docente. Revista de Educación, 362, 380-401.

Oliver, K.M., \& Corn, J. (2008). Student-reported differences in technology use and skills after the implementation of one-to-one computing. Educational Media International, 45,215-229. https://doi.org/10.1080/09523980802284333

Prieto, R.; Smok, C. \& Rojas, M. (2011). Blog Experiences: Compared Placenta. International Journal of Morphology, 29(2):432-437. https://doi.org/10.4067/S0717-95022011000200022

Raikos, A., \& Waidyasekara, P. (2014). How useful is YouTube in learning heart anatomy?. Anatomical Sciences Education, 7(1), 12-18. https://doi.org/10.1002/ase.1361

Ravi, K. S. (2020). Dead body management in times of Covid-19 and its potential impact on the availability of cadavers for medical education in India. Anatomical Sciences Education, 13(3), 316-317. https://doi.org/10.1002/ase.1962

Srinivasan, D. K. (2020). Medical students' perceptions and an anatomy teacher's personal experience using an elearning platform for tutorials during the Covid-19 crisis. Anatomical Sciences Education, 13(3), 318-319. https://doi.org/10.1002/ase.1970

Thomas, A., \& Stratton, G. (2006). What we are really doing with ICT in physical education: A national audit of equipment, use, teacher attitudes, support, and training. British Journal of Educational Technology, 37, 617632. https://doi.org/10.1111/j.1467-8535.2006.00520.x 